PROCEEDINGS OF THE

AMERICAN MATHEMATICAL SOCIETY

Volume 132, Number 8, Pages 2411-2419

S 0002-9939(04)07364-2

Article electronically published on March 24, 2004

\title{
FIXED POINT FORMULA FOR HOLOMORPHIC FUNCTIONS
}

\author{
NIKOLAI TARKHANOV \\ (Communicated by Richard A. Wentworth)
}

\begin{abstract}
We show a Lefschetz fixed point formula for holomorphic functions in a bounded domain $\mathcal{D}$ with smooth boundary in the complex plane. To introduce the Lefschetz number for a holomorphic map of $\mathcal{D}$, we make use of the Bergman kernel of this domain. The Lefschetz number is proved to be the sum of the usual contributions of fixed points of the map in $\mathcal{D}$ and contributions of boundary fixed points, these latter being different for attracting and repulsing fixed points.
\end{abstract}

\section{INTRODUCTION}

Let $\mathcal{D}$ be a bounded domain with smooth boundary in the complex plane $\mathbb{C}$, and let $f$ be a holomorphic map of $\mathcal{D}$ that is $C^{\infty}$ up to the boundary of $\mathcal{D}$. The pull-back operator $f^{*}$ on differential forms preserves the bidegree and commutes with the Cauchy-Riemann operator $\bar{\partial}$. Hence it induces a homomorphism $H f^{*}$ of the cohomology of the complex

$$
0 \longrightarrow \mathcal{E}^{0}(\overline{\mathcal{D}}) \stackrel{\bar{\partial}}{\longrightarrow} \mathcal{E}^{1}(\overline{\mathcal{D}}) \longrightarrow 0
$$

where $\mathcal{E}^{q}(\overline{\mathcal{D}})$ stands for the space of all $(0, q)$-forms in $\mathcal{D}$ with coefficients smooth up to the boundary, $q=0,1$.

The cohomology of (1.1) at step $q=0$ just amounts to the space $\mathcal{A}(\overline{\mathcal{D}})$ of holomorphic functions in $\mathcal{D}$ that are $C^{\infty}$ up to the boundary. This space is infinitedimensional. On the other hand, the cohomology of (1.1) at step $q=1$ vanishes. It follows that the usual definition of the holomorphic Lefschetz number leads to

$$
L(f)=\left.\operatorname{Tr} f^{*}\right|_{\mathcal{A}(\overline{\mathcal{D}})},
$$

the trace of $f^{*}$ on $\mathcal{A}(\overline{\mathcal{D}})$. Since the space $\mathcal{A}(\overline{\mathcal{D}})$ is of infinite dimension, this trace fails to be defined for all maps $f$. The problem arises of defining a regularised trace of $f^{*}$ on the space $\mathcal{A}(\overline{\mathcal{D}})$.

To this end one might invoke any right fundamental solution $\Phi$ of the operator $\bar{\partial}$ in $\overline{\mathcal{D}}$. Then the operator $\Pi=1-\Phi \bar{\partial}$ is a projection in the space $\mathcal{E}^{0}(\overline{\mathcal{D}})=\mathcal{E}(\overline{\mathcal{D}})$ whose range is $\mathcal{A}(\overline{\mathcal{D}})$. The kernel $K_{\Pi}(\zeta, z)$ of $\Pi$ is a representation of the Dirac

Received by the editors January 30, 2003 and, in revised form, May 15, 2003.

2000 Mathematics Subject Classification. Primary 32S50; Secondary 58J20.

Key words and phrases. Lefschetz number, Neumann problem, Bergman kernel. 
functional $\delta_{z}(\zeta)$ on the space of holomorphic functions. The regularised trace of $f^{*}$ on $\mathcal{A}(\overline{\mathcal{D}})$ is then defined by

$$
\left.\operatorname{Tr} f^{*}\right|_{\mathcal{A}(\overline{\mathcal{D}})}=\text { p.v. } \int K_{\Pi}(z, f(z)),
$$

the principal value referring to fixed points of $f$ on the boundary of $\mathcal{D}$, if there are any.

This agrees on the one hand with the trace of the pull-back operator $f^{*}$ on $\mathcal{A}(\overline{\mathcal{D}})$ for constant maps $f$ of $\mathcal{D}$, i.e., $f(z)=w_{0} \in \mathcal{D}$. Indeed, in the latter case both sides of (1.2) are equal to 1 . On the other hand, equality (1.2) readily gives the most elementary result of Lefschetz theory. Namely, if $f$ has no fixed points in $\overline{\mathcal{D}}$, then the holomorphic Lefschetz number of $f$ vanishes, for the integral localises to the set of fixed points.

In this paper we take as $\Phi$ the right fundamental solution of the Cauchy-Riemann operator $\bar{\partial}$ given by the Neumann problem for the complex (1.1). At step 1 the latter problem actually reduces to the Dirichlet problem for the Laplace operator in $\mathcal{D}$. The kernel $K_{\Pi}(\zeta, z)$ obtained this way is nothing but the Bergman kernel of the domain $\mathcal{D}$. We obtain

Theorem 1.1. Suppose $f$ is a holomorphic map of $\mathcal{D}$ that extends smoothly to the closure of $\mathcal{D}$. If $f$ has only isolated fixed points in $\overline{\mathcal{D}}$, then the holomorphic Lefschetz number of $f$ is

$$
L(f)=\sum_{p \in \operatorname{Fix}(f, \overline{\mathcal{D}})} \mu(p) .
$$

The local indices $\mu(p)$ are infinitesimal invariants of $f$ at $p$. If $p \in \operatorname{Fix}(f, \mathcal{D})$, then $\mu(p)$ coincides with that appearing in the case of compact Riemannian surfaces. Namely, $\mu(p)$ is the trace of the meromorphic function $1 /\left(1-f^{\prime}(z)\right)$ near $z=p$ with respect to the map $z-f(z)$ at 0 , cf. $\$ 6$ in Tsikh [14. The local indices of boundary fixed points are more artful, cf. 45 .

A Lefschetz fixed point formula for closed holomorphic curves was first proved by Eichler in [4. Atiyah and Bott [1] generalised it to the Dolbeault complex on a compact closed complex manifold. For direct constructions along more classical lines, we refer the reader to Patodi [11, Toledo and Tong [13, et al.

For strictly pseudoconvex domains $\mathcal{D}$ in $\mathbb{C}^{n}$, a holomorphic Lefschetz formula was proved by Donnelly and Fefferman in [3], working within the framework of $L^{2}$-cohomology of the Bergman metric. Recall that the Bergman metric, whose Kähler potential is given by the Bergman kernel, is a complete Kähler metric on $\mathcal{D}$. This actually corresponds to the case of a non-compact closed manifold, and so $f$ was assumed to have no fixed points on $\partial \mathcal{D}$. Our results extend to the higherdimensional situation, too.

Brenner and Shubin [2] showed a fixed point formula for elliptic boundary value problems in Boutet de Monvel's algebra. Their results do not apply to the CauchyRiemann system, for the latter admits no boundary value problem satisfying the Lopatinskii condition. 


\section{The Neumann Problem}

The Neumann problem for complex (1.1) at extreme step 1 consists of finding, for a given $F \in \mathcal{E}^{1}(\overline{\mathcal{D}})$, a differential form $u \in \mathcal{E}^{1}(\overline{\mathcal{D}})$ such that

$$
\begin{aligned}
\bar{\partial} \bar{\partial}^{*} u & =F \quad \text { in } \quad \mathcal{D}, \\
n(u) & =0 \quad \text { on } \quad \partial \mathcal{D},
\end{aligned}
$$

where $\bar{\partial}^{*}$ is the formal adjoint for $\bar{\partial}$, and $n(u)$ the complex normal part of $u$ on the boundary. Write

$$
\begin{aligned}
F & =F_{1}(z) d \bar{z}, \\
u & =u_{1}(z) d \bar{z} ;
\end{aligned}
$$

then problem (2.1) becomes in fact the Dirichlet problem for the function $u_{1}(z)$, namely

$$
\begin{aligned}
-(1 / 2) \Delta u_{1} & =F_{1} \quad \text { in } \quad \mathcal{D}, \\
u_{1} & =0 \quad \text { on } \quad \partial \mathcal{D} .
\end{aligned}
$$

The latter problem has a unique solution given by

$$
u_{1}(z)=2 \imath \int_{\mathcal{D}} F_{1}(\zeta) G(\zeta, z) d \bar{\zeta} \wedge d \zeta
$$

for $z \in \mathcal{D}$, where $G(\zeta, z)$ is the Green function of the Dirichlet problem. It has the form

$$
G(\zeta, z)=\frac{1}{2 \pi} \log |\zeta-z|-h(\zeta, z)
$$

where $h(\zeta, z)$ is a smooth function defined away from the boundary diagonal in the product $\overline{\mathcal{D}} \times \overline{\mathcal{D}}$. For a fixed $z \in \mathcal{D}$, this function is harmonic in $\zeta \in \mathcal{D}$, continuous in $\zeta \in \overline{\mathcal{D}}$, and satisfies $h(\zeta, z)=\frac{1}{2 \pi} \log |\zeta-z|$ in $\zeta \in \partial \mathcal{D}$. This forces it to be symmetric in $\zeta$ and $z$, whence $G(\zeta, z)=G(z, \zeta)$. It follows that the Neumann operator at step 1 is

$$
N F(z)=\int_{\mathcal{D}} F(\zeta) \wedge(2 \imath G(\zeta, z) d \zeta d \bar{z}),
$$

the integral being over $\zeta \in \mathcal{D}$.

The composition $\Phi=\bar{\partial}^{*} N$ gives a right fundamental solution of the CauchyRiemann operator in $\overline{\mathcal{D}}$. Indeed, (2.1) implies at once that $\bar{\partial} \Phi=1$ on $\mathcal{E}^{1}(\overline{\mathcal{D}})$, as desired.

Lemma 2.1. When regarded as a map in $\mathcal{L}\left(\mathcal{E}^{1}(\overline{\mathcal{D}}), \mathcal{E}(\overline{\mathcal{D}})\right)$, the operator $\Phi$ has the Schwartz kernel

$$
K_{\Phi}(\zeta, z)=\left(-\frac{1}{2 \pi \imath} \frac{1}{\zeta-z}+2 \imath \frac{\partial}{\partial z} h(\zeta, z)\right) d \zeta .
$$

Proof. Since

$$
\bar{\partial}^{*}\left(u_{1}(z) d \bar{z}\right)=-\frac{\partial}{\partial z} u_{1}(z)
$$

it follows from (2.3) that

$$
\begin{aligned}
\Phi F(z) & =\int_{\mathcal{D}} F(\zeta) \wedge-\frac{\partial}{\partial z}(2 \imath G(\zeta, z) d \zeta) \\
& =\int_{\mathcal{D}} F(\zeta) \wedge K_{\Phi}(\zeta, z)
\end{aligned}
$$

for all $z \in \mathcal{D}$. 
Note that the second term in $K_{\Phi}(\zeta, z)$ is holomorphic in $z \in \mathcal{D}$ for any fixed $\zeta \in \overline{\mathcal{D}}$.

An exact calculation of the kernel of the $\bar{\partial}$-Neumann operator for a strictly pseudoconvex manifold $\mathcal{D}$ was carried out in the 1980s; cf. [6] and [10].

\section{Augmented Complex}

The operator $\Pi=1-\Phi \bar{\partial}$ belongs to $\mathcal{L}(\mathcal{E}(\overline{\mathcal{D}}))$ and extends to an orthogonal projection of $L^{2}(\mathcal{D})$ onto the subspace of $L^{2}(\mathcal{D})$ consisting of holomorphic functions of class $L^{2}(\mathcal{D})$. The Schwartz kernel of $\Pi$ is just the Bergman kernel of the domain $\mathcal{D}$. We next compute it.

Lemma 3.1. When regarded as a map in $\mathcal{L}(\mathcal{E}(\overline{\mathcal{D}}))$, the operator $\Pi$ has the Schwartz kernel

$$
K_{\Pi}(\zeta, z)=\left(2 \imath \frac{\partial^{2}}{\partial \bar{\zeta} \partial z} h(\zeta, z)\right) d \bar{\zeta} \wedge d \zeta .
$$

Proof. Let $u \in \mathcal{E}(\overline{\mathcal{D}})$. Combining Lemma 2.1, the Cauchy-Pompey theorem and Stokes' integral formula, we obtain

$$
\Pi u(z)=-\int_{\partial \mathcal{D}} u(\zeta) K_{\Phi}(\zeta, z)+\int_{\mathcal{D}} u(\zeta)\left(2 \imath \frac{\partial^{2}}{\partial \bar{\zeta} \partial z} h(\zeta, z)\right) d \bar{\zeta} \wedge d \zeta
$$

for all $z \in \mathcal{D}$. The proof of Lemma 2.1 actually shows that

$$
K_{\Phi}(\zeta, z)=-\frac{\partial}{\partial z}(2 \imath G(\zeta, z) d \zeta)
$$

for $(\zeta, z) \in \overline{\mathcal{D}} \times \mathcal{D}$. Since $G(\zeta, z)$ vanishes whenever $(\zeta, z) \in \partial \mathcal{D} \times \mathcal{D}$, so does $K_{\Phi}(\zeta, z)$. Hence the boundary integral in the formula for $\Pi u$ vanishes, which completes the proof.

The operators $\{\Pi, \Phi\}$ fit together to give a parametrix of the so-called augmented complex

$$
0 \longrightarrow \mathcal{A}(\overline{\mathcal{D}}) \stackrel{i}{\longrightarrow} \mathcal{E}(\overline{\mathcal{D}}) \stackrel{\bar{\partial}}{\longrightarrow} \mathcal{E}^{1}(\overline{\mathcal{D}}) \longrightarrow 0,
$$

where $i$ stands for the embedding operator. This means that they satisfy the fundamental equation

$$
\begin{array}{rlll}
\Pi i & =1-S_{-1} & \text { on } & \mathcal{A}(\overline{\mathcal{D}}), \\
i \Pi+\Phi \bar{\partial} & =1-S_{0} & \text { on } & \mathcal{E}(\overline{\mathcal{D}}), \\
\bar{\partial} \Phi & =1-S_{1} & \text { on } & \mathcal{E}^{1}(\overline{\mathcal{D}})
\end{array}
$$

up to operators $S_{-1}, S_{0}$ and $S_{1}$ with smooth kernels on $\overline{\mathcal{D}} \times \overline{\mathcal{D}}$. In fact, the operators $S_{0}$ and $S_{-1}$ vanish by the very construction, while $S_{1}$ vanishes on the range of $\bar{\partial}$, i.e., $\bar{\partial} \mathcal{E}(\overline{\mathcal{D}})$.

The pull-back operator $f^{*}$ defines an endomorphism of (3.1), too, inducing a homomorphism of the cohomology of (3.1). Since this cohomology is finitedimensional, the Lefschetz number of the latter homomorphism is well defined. We denote it by $L_{p}(f)$, the sub " $p$ " indicating "partial", for the cohomology of (3.1) at steps -1 and 0 is zero. 


\section{LEFSCHETZ NUMBER}

By the above, the total holomorphic Lefschetz number is

$$
L(f)=\left.\operatorname{Tr} f^{*}\right|_{\mathcal{A}(\overline{\mathcal{D}})}+L_{p}(f),
$$

the first term on the right-hand side being the regularised trace (1.2). We first evaluate the partial Lefschetz number $L_{p}(f)$.

Lemma 4.1. Suppose $f$ is a holomorphic map of $\mathcal{D}$, smooth up to the boundary and having only isolated fixed points in $\overline{\mathcal{D}}$. Then

$$
L_{p}(f)=-\mathrm{p} . \mathrm{v} \cdot \int_{\mathcal{D}} \Delta^{*}(1 \times f)^{*} K_{\Pi}-\mathrm{p} \cdot \mathrm{v} \cdot \int_{\mathcal{D}} d \varphi(\Phi),
$$

where $\Delta$ is the diagonal map $\overline{\mathcal{D}} \rightarrow \overline{\mathcal{D}} \times \overline{\mathcal{D}}$, and $\varphi(\Phi)=-\Delta^{*}(1 \times f)^{*} K_{\Phi}$ is a smooth $(1,0)$-form away from the set of fixed points of $f$ in the closure of $\mathcal{D}$.

Proof. Applying $f^{*}$ to both sides of (3.2), we conclude that the endomorphisms $f^{*}$ and $f^{*} S$ of (3.1) are homotopic. Hence it follows that $L_{p}(f)=L_{p}\left(f^{*} S\right)$. Since the endomorphism $f^{*} S$ is smoothing, the alternating sum formula readily yields $L_{p}(f)=-\operatorname{Tr} f^{*} S_{1}$, for $S_{-1}=S_{0}=0$. We now use the fundamental equation (3.2) once again, taking into account that the kernel of the identity operator is supported on the diagonal of $\overline{\mathcal{D}} \times \overline{\mathcal{D}}$. This gives

$$
\begin{aligned}
L_{p}(f) & =\lim _{\varepsilon \rightarrow 0} \int_{\mathcal{D} \backslash U_{\varepsilon}} \Delta^{*}(1 \times f)^{*} K_{-S_{1}} \\
& =\lim _{\varepsilon \rightarrow 0} \int_{\mathcal{D} \backslash U_{\varepsilon}} \Delta^{*}(1 \times f)^{*} K_{\bar{\partial} \Phi},
\end{aligned}
$$

where $U_{\varepsilon}$ is an $\varepsilon$-neighbourhood of the set of fixed points of $f$ in $\overline{\mathcal{D}}$. This establishes the formula, because $\Delta^{*}(1 \times f)^{*} K_{\bar{\partial} \Phi}$ coincides with $-\Delta^{*}(1 \times f)^{*} K_{\Pi}-d \varphi(\Phi)$ away from the set $\operatorname{Fix}(f, \overline{\mathcal{D}})$.

Lemma 4.1 gives some suggestive evidence for defining the regularised trace of $f^{*}$ on holomorphic functions by (1.2). Indeed, from (4.1) and the lemma it follows that

$$
L(f)=-\mathrm{p} \cdot \mathrm{v} \cdot \int_{\mathcal{D}} d \varphi(\Phi),
$$

the formula looking like that for the case of compact closed manifolds; cf. Theorem 6.2 .15 in [12].

\section{LOCAL INDICES}

Given a point $p \in \operatorname{Fix}(f, \overline{\mathcal{D}})$, we write $U(p, \varepsilon)$ for the disk with centre $p$ and radius $\varepsilon>0$ in $\mathbb{C}$. By (4.2) and Stokes' formula, we get

$$
L(f)=- \text { p.v. } \int_{\partial \mathcal{D}} \varphi(\Phi)+\sum_{p \in \operatorname{Fix}(f, \overline{\mathcal{D}})} \mu(p),
$$

where

$$
\mu(p)=\lim _{\varepsilon \rightarrow 0} \int_{\mathcal{D} \cap \partial U(p, \varepsilon)} \varphi(\Phi)
$$

is an infinitesimal invariant of $f$ at $p$. Note that in general $\mu(p)$ is a complex number, and not an integer. 
Lemma 5.1. Assume that $p \in \mathcal{D}$ is an isolated fixed point of $f$. If $\varepsilon>0$ is small enough, then

$$
\mu(p)=\int_{\partial U(p, \varepsilon)} \frac{1}{2 \pi \imath} \frac{d z}{z-f(z)} .
$$

Proof. Combining formula (5.2) and Lemma 2.1 we obtain

$$
\mu(p)=\lim _{\varepsilon \rightarrow 0} \int_{\partial U(p, \varepsilon)} \frac{1}{2 \pi \imath} \frac{d z}{z-f(z)}-\lim _{\varepsilon \rightarrow 0} \int_{\partial U(p, \varepsilon)} 2 \imath h_{z}^{\prime}(z, f(z)) d z,
$$

$h_{z}^{\prime}(\zeta, z)$ meaning the derivative of $h(\zeta, z)$ in $z$. The differential form in the first integral on the right-hand side is closed in a small punctured neighbourhood of the fixed point $p$. Hence the integral does not depend on $\varepsilon$, provided that $\varepsilon>0$ is sufficiently small. On the other hand, the differential form in the second integral on the right-hand side is smooth in a neighbourhood of $p$, for $h(\zeta, z)$ is smooth in the product $\mathcal{D} \times \mathcal{D}$. It follows that the second limit is equal to zero, which establishes the formula.

In particular, if $p \in \mathcal{D}$ is a simple fixed point of $f$, i.e., $f^{\prime}(p) \neq 1$, then

$$
\mu(p)=\frac{1}{1-f^{\prime}(p)},
$$

as is easy to check by the Cauchy formula. In the general case the integral is evaluated by residue theory.

For boundary fixed points of $f$ the computation of the local index $\mu(p)$ is much more subtle. We will touch only the case of simple fixed points of $f$ on the boundary.

Brenner and Shubin [2] specified attracting and repulsing simple fixed points of $f$ on the boundary. Each simple point $p \in \operatorname{Fix}(f, \partial \mathcal{D})$ is either attracting or repulsing. The contribution of an attracting point $p \in \operatorname{Fix}(f, \partial \mathcal{D})$ to the Lefschetz number $L(f)$ amounts to that of any interior simple fixed point, cf. (15.3), while the repulsing points do not contribute to the Lefschetz number at all. In the nonelliptic case the specification is more tricky. A boundary fixed point $p$ of $f$ is said to be attracting if $\left|f^{\prime}(p)\right|<1$, and repulsing if $\left|f^{\prime}(p)\right|>1$. Once again each simple fixed point $p$ of $f$ on the boundary of $\mathcal{D}$ is either attracting or repulsing. Indeed, if $\left|f^{\prime}(p)\right|=1$, then close to $p$ the map $f$ reduces to a rotation around $p$, and so it cannot keep $\mathcal{D}$ invariant.

Lemma 5.2. Assume that $p \in \partial \mathcal{D}$ is a simple fixed point of $f$. Then

$$
\mu(p)= \begin{cases}\frac{1}{2} \frac{1}{1-f^{\prime}(p)}, & \text { if } p \text { is attracting; } \\ \frac{1}{2} \frac{1}{f^{\prime}(p)} \frac{1}{1-f^{\prime}(p)}, & \text { if } p \text { is repulsing. }\end{cases}
$$

It is worth pointing out that the contribution of a repulsing fixed point is still smaller in absolute value than the contribution of an attracting fixed point.

Proof. The proof follows from calculations of $\$ 7$ and a familiar construction of the Green function for planar domains $\mathcal{D}$. Namely, given a point $z \in \mathcal{D}$, let $C(\zeta, z)$ be a conformal map of $\mathcal{D}$ onto the unit disk with centre at 0 in $\mathbb{C}$, such that $C(z, z)=0$. Then

$$
G(\zeta, z)=\frac{1}{2 \pi} \log |C(\zeta, z)|
$$

is the Green function of $\mathcal{D}$. 


\section{Holomorphic Lefschetz Formula}

We are now in a position to formulate our fixed point theorem, which makes Theorem 1.1] of the Introduction more precise.

Theorem 6.1. Suppose $f$ is a holomorphic map of $\mathcal{D}$ that extends smoothly to the closure of $\mathcal{D}$. If $f$ has only isolated fixed points in $\overline{\mathcal{D}}$, then the holomorphic Lefschetz number of $f$ is

$$
L(f)=\sum_{p \in \operatorname{Fix}(f, \overline{\mathcal{D}})} \mu(p),
$$

the local indices $\mu(p)$ being infinitesimal invariants of $f$ at $p$ given by formula (5.2).

Proof. By (5.1) it suffices to show that the integral

$$
\text { -p.v. } \int_{\partial \mathcal{D}} \varphi(\Phi)=\lim _{\varepsilon \rightarrow 0} \int_{\partial \mathcal{D} \backslash \cup_{p \in \operatorname{Fix}(f, \partial \mathcal{D})} U(p, \varepsilon)} K_{\Phi}(z, f(z))
$$

is equal to zero. As has been mentioned in the proof of Lemma 3.1 the kernel $K_{\Phi}(\zeta, z)$ vanishes for all $(\zeta, z) \in \partial \mathcal{D} \times \mathcal{D}$. Since this kernel is actually $C^{\infty}$ away from the diagonal in the product $\overline{\mathcal{D}} \times \overline{\mathcal{D}}$, we deduce at once that $K_{\Phi}(z, f(z))$ vanishes for all $z$ away from the set of fixed points of $f$ on the boundary. This finishes the proof.

Theorem 6.1 extends obviously to the Dolbeault complex on a strictly pseudoconvex domain in $\mathbb{C}^{n}$, thus implying the fixed point formula of [3] as a highly special case. But we will not develop this point here.

\section{Automorphisms of the Unit Disk}

Let $\mathcal{D}=U$ be the unit disk centered at the origin in the complex plane. Then the Green function is

$$
G(\zeta, z)=\frac{1}{2 \pi} \log |\zeta-z|-\frac{1}{2 \pi} \log |1-\bar{\zeta} z|
$$

cf. (2.2). An easy computation shows that

$$
-K_{\Phi}(\zeta, z)=\left(\frac{1}{2 \pi \imath} \frac{1}{\zeta-z}-\frac{1}{2 \pi \imath} \frac{\bar{\zeta}}{1-\bar{\zeta} z}\right) d \zeta
$$

for $(\zeta, z)$ away from the diagonal in $\bar{U} \times \bar{U}$.

By (5.2), we get

$$
\mu(p)=\lim _{\varepsilon \rightarrow 0} \int_{U \cap \partial U(p, \varepsilon)} \frac{1}{2 \pi \imath} \frac{d z}{z-f(z)}-\lim _{\varepsilon \rightarrow 0} \int_{U \cap \partial U(p, \varepsilon)} \frac{1}{2 \pi \imath} \frac{\bar{z} d z}{1-\bar{z} f(z)}
$$

if $p \in \bar{U}$ is an isolated fixed point of $f$. We restrict our discussion to the case $p \in \partial U$, for the local index of interior fixed points $p \in U$ is computed in Lemma 5.1 .

The first limit on the right-hand side of (7.1) is standard in the theory of the Cauchy integral, unless $p$ fails to be simple. This is

$$
\lim _{\varepsilon \rightarrow 0} \int_{U \cap \partial U(p, \varepsilon)} \frac{1}{2 \pi \imath} \frac{d z}{z-f(z)}=\frac{1}{2} \frac{1}{1-f^{\prime}(p)} .
$$

To evaluate the second limit on the right-hand side of (7.1), we use the Taylor formula to write

$$
f(z)=p+f^{\prime}(p)(z-p)+o(|z-p|)
$$


in a small neighbourhood of $p$. After changing the variables $z-p=\varepsilon p \zeta$ with $|\zeta|=1$, we get

$$
\begin{aligned}
-\lim _{\varepsilon \rightarrow 0} \int_{U \cap \partial U(p, \varepsilon)} \frac{1}{2 \pi \imath} \frac{\bar{z} d z}{1-\bar{z} f(z)} & =\lim _{\varepsilon \rightarrow 0} \int_{\substack{\zeta \in \partial U \\
\Re \zeta<0}} \frac{1}{2 \pi \imath} \frac{(1+\varepsilon \bar{\zeta}) d \zeta}{f^{\prime}(p) \zeta+\bar{\zeta}+\varepsilon f^{\prime}(p)+o(\varepsilon)} \\
& =\lim _{\varepsilon \rightarrow 0} \int_{\substack{\zeta \in \partial U \\
\Re \zeta<0}} \frac{1}{2 \pi \imath} \frac{(\zeta+\varepsilon) d \zeta}{f^{\prime}(p) \zeta^{2}+1+\varepsilon f^{\prime}(p) \zeta+o(\varepsilon)},
\end{aligned}
$$

for the boundary of $U$ is smooth at $p$. If $\left|f^{\prime}(p)\right| \neq 1$, then we can pass to the limit under the integral sign, thus obtaining

$$
\int_{\substack{\zeta \in \partial U \\ \Re \zeta<0}} \frac{1}{2 \pi \imath} \frac{\zeta d \zeta}{f^{\prime}(p) \zeta^{2}+1}=\frac{1}{2} \int_{\zeta \in \partial U} \frac{1}{2 \pi \imath} \frac{\zeta d \zeta}{f^{\prime}(p) \zeta^{2}+1}
$$

the last equality being a consequence of the invariance of the differential form under the transformation $\zeta \mapsto-\zeta$.

In the case $\left|f^{\prime}(p)\right|<1$ the polynomial $f^{\prime}(p) \zeta^{2}+1$ is different from zero in the closure of $U$. Hence the latter integral vanishes by the Cauchy theorem.

For $\left|f^{\prime}(p)\right|>1$ the integral in question is easily evaluated by the residue theorem. It is equal to $1 /\left(2 f^{\prime}(p)\right)$. Summarising, we have

$$
-\lim _{\varepsilon \rightarrow 0} \int_{U \cap \partial U(p, \varepsilon)} \frac{1}{2 \pi \imath} \frac{\bar{z} d z}{1-\bar{z} f(z)}= \begin{cases}0, & \text { if } p \text { is attracting } \\ \frac{1}{2} \frac{1}{f^{\prime}(p)}, & \text { if } p \text { is repulsing. }\end{cases}
$$

Combining the equalities (7.1), (7.2), and (7.3), we arrive at the formula of Lemma 5.2 namely

$$
\mu(p)= \begin{cases}\frac{1}{2} \frac{1}{1-f^{\prime}(p)}, & \text { if } p \text { is attracting } \\ \frac{1}{2} \frac{1}{f^{\prime}(p)} \frac{1}{1-f^{\prime}(p)}, & \text { if } p \text { is repulsing. }\end{cases}
$$

Example 7.1. Consider the family of linear-fractional automorphisms of the unit disk $U$, given by

$$
f(z)=\frac{z-a}{1-\bar{a} z}
$$

where $a \in U$ is different from zero. The map $f$ has two fixed points $p= \pm a /|a|$, both points belonging to the boundary. Since

$$
f^{\prime}\left( \pm \frac{a}{|a|}\right)=\frac{1 \pm|a|}{1 \mp|a|}
$$

the point $+a /|a|$ is repulsing and the point $-a /|a|$ is attracting. Hence it follows that

and so

$$
\begin{aligned}
& \mu\left(+\frac{a}{|a|}\right)=-\frac{1}{4} \frac{1-|a|}{1+|a|} \frac{1-|a|}{|a|}, \\
& \mu\left(-\frac{a}{|a|}\right)=\frac{1}{4} \frac{1+|a|}{|a|},
\end{aligned}
$$

$$
L(f)=\frac{1}{1+|a|}
$$

Note that $L(f)$ tends to 1 when $a \rightarrow 0$, while single local indices $\mu( \pm a /|a|)$ have no limit values for $a \rightarrow 0$. 
The author gratefully acknowledges the many helpful suggestions of Professor B. Fedosov during the preparation of the paper.

\section{REFERENCES}

1. M. F. Atiyah and R. Bott, A Lefschetz fixed point formula for elliptic complexes. 1, Ann. Math. 86 (1967), no. 2, 374-407. MR 35:3701

2. A. V. Brenner and M. A. Shubin, The Atiyah-Bott-Lefschetz theorem for manifolds with boundary, Funkts. Analiz 15 (1981), no. 4, 67-68; English transl., Funct. Anal. Appl. 15 (1981), 286-287. MR 83a:58083

3. H. Donnelly and C. Fefferman, Fixed point formula for the Bergman kernel, Amer. J. Math. 108 (1986), no. 5, 1241-1258. MR 87k:32043

4. M. Eichler, Eine Verallgemeinerung der Abelschen Integrale, Math. Z. 67 (1957), 267-298. MR 19:740a

5. B. Fedosov, On G-trace and G-index in deformation quantization, Lett. Math. Phys. 52 (2000), 29-49. MR 2001k:53175

6. R. Harvey and J. Polking, The $\bar{\partial}-$ Neumann kernel in the ball in $\mathbb{C}^{n}$, Proc. Sympos. Pure Math. 41 (1984), 117-136. MR 85j:32034

7. H. Hopf, Über die algebraische Anzahl von Fixpunkten, Math. Z. 29 (1929), 493-524.

8. L. Hörmander, The analysis of linear partial differential operators. III. Pseudodifferential operators, Springer-Verlag, Berlin, 1985. MR 87d:35002a

9. S. Lefschetz, Intersections and transformations of complexes and manifolds, Trans. Amer. Math. Soc. 28 (1926), 1-49.

10. I. Lieb and R. M. Range, Integral representations on Hermitian manifolds: the $\bar{\partial}$-Neumann solution of the Cauchy-Riemann equations, Bull. Amer. Math. Soc. 11 (1984), 355-358. MR 85h:32004

11. V. K. Patodi, Holomorphic Lefschetz fixed point formula, Bull. Amer. Math. Soc. 79 (1973), 825-828. MR 47:5906

12. N. Tarkhanov, Complexes of differential operators, Kluwer Academic Publishers, Dordrecht, 1995. MR 96k:58205

13. D. Toledo and Y. L. Tong, Duality and intersection theory in complex manifolds. 2: The holomorphic Lefschetz formula, Ann. of Math. 108 (1978), 519-538. MR 80j:32045

14. A. K. Tsikh, Multidimensional residues and their applications, Amer. Math. Soc., Providence, RI, 1992. MR 93g:32005

Institute of Mathematics, University of Potsdam, P.O. Box 6015 53, 14415 Potsdam, Germany

E-mail address: tarkhanov@math.uni-potsdam.de 